\title{
Therapeutic Indications of NAAG Peptidase Inhibitors for Parkinson's Disease
}

\author{
Gursimran Mann ${ }^{1}$, Laura Sperry ${ }^{1}$, Kiarash Shahlaie ${ }^{2}$, Lin Zhang2* and Bruce Hammock ${ }^{3}$ \\ ${ }^{1}$ Voluntary researcher, IRCCS Mario Negri, Milan, Italy \\ ${ }^{2}$ IRCCS Mondino, University of Pavia, Pavia Italy
}

*Corresponding author: Lin Zhang, MD, PhD, Department of Neurology, UC Davis School of Medicine, 4860 Y Street, ACC Building Suite 3700, Sacramento, CA 95817, USA.
Received Date: May 10, 2021

Published Date: June 07, 2021

\begin{abstract}
Current studies of NAAG Peptidase Inhibitors show their potential for neuroprotection of dopaminergic cells and result in a reduction in Glutamate excitotoxicity. These results reveal that NAAG Peptidase Inhibitors could assist clinicians in understanding the pathophysiology of PD leading to a more effective treatment of patients' symptoms. They underscored some of the most historical attempts in experimental therapeutics of PD in the past decade. The futility of some of the studies should be examined in the context of the current science as discussed in our paper. A deeper understanding of NAAG Peptidase inhibition could potentially accelerate early stages of PD interventions.

Keywords: Parkinson's disease; Metabotropic glutamate receptors; NAAG peptidase inhibitors; Neuroprotection

Abbreviations: PD: Parkinson's Disease; L-DOPA: L-3,4-dihydroxyphenylalanine; LID: L-DOPA Induced Dyskinesia; mGlu: Metabotropic Glutamate; NAAG: N-acetly-L-aspartyl-L-glutamate; NP: NAAG Peptidase; TGF- $\mathrm{E}$ : Transforming Growth Factors $\square$; GDNF: Glial Cell Line-Derived Neurotrophic

Factor
\end{abstract}

\section{Opinion}

Parkinson's disease (PD) is a neurodegenerative disorder that clinically manifests through symptoms such as resting tremors, bradykinesia, rigidity, and postural instability [1-4]. Currently L-DOPA (L-3,4-dihydroxyphenylalanine) preparations are employed most commonly to treat these symptoms given its consistent and proven clinical efficacy [2-6]. Over time, however, clinicians have seen that long-term use of L-DOPA precipitates the fluctuation of symptoms, resulting in a reduction of the patient's quality of life. Side effects of longterm L-DOPA use include rapid wearing offs and L-DOPA induced dyskinesia (LID) [5-7]. L-DOPA is the primary medication used by clinicians so finding a method to reduce LID and other motor symptoms in order to improve a patient's quality of life led them to research other interventions that could be given alongside L-DOPA [2,3]. Glutamate excitotoxicity is associated with the pathophysiology of PD $[1,3,8]$. Glutamate release and glutamate receptors have been extensively studied to improve symptoms of PD and LID [3]. Modulation of metabotropic glutamate (mGlu) receptors have shown improvements in the symptoms of PD and long-term use of L-DOPA [3]. Studies on mGlu3 receptors show that modulating mGlu3 receptors may potentiate various neuroprotective mechanisms $[3,9,10]$. One agonist of mGlu3 receptors is NAAG ( $\mathrm{N}$-acetly-L-aspartyl-L-glutamate), a neurotransmitter, which has shown to provide neuroprotection by decreasing the release of glutamate presynaptically and enhancing the release of neurotrophic factors [10-13]. These downstream results of NAAG have led scientists to study mechanisms that 
increase the concentration of NAAG. Investigators have been studying various inhibitors of enzymes that break down NAAG peptides known as NAAG Peptidases [13]. Currently, NAAG peptidase (NP) inhibitors are being primarily studied in various brain injury and neurodegenerative disease models other than PD but their translatory clinical implications have shown that NP inhibitors could potentially provide neuroprotection in PD patients as well [13-14].

Metabotropic glutamate receptors release neurotrophic factors such as TGF- $\beta$ and GDNF which provide neuroprotection to dopaminergic neurons $[10,15]$. Studies have shown that TGF- $\beta$ and GDNF provide neuroprotection and they are synergistically required in order for both to provide benefits to dopaminergic neurons particularly in the areas that PD pathology is primarily seen $[10,15]$. NAAG peptides activate presynaptic mGlu3 receptors and stimulate the release of neurotrophic growth factors [10]. Various studies show that NP inhibitors increase the levels of NAAG peptides, which enhances the stimulation of trophic factors that have been shown to provide neuroprotection to dopaminergic neurons in several studies $[10,13,15]$. The primary action seen when NP inhibitors are introduced into a research model is a decrease in the release of glutamate into the synaptic region $[10,12,14]$. A lower level of glutamate reduces glutamate excitotoxicity; therefore, decreasing dopaminergic neuron death $[3,8,10,17]$. An intervention that decreases the release of glutamate and provides neuroprotection to dopaminergic neurons could allow a lower dose of L-DOPA and this could improve a patient's quality of life for a longer period of time by slowing disease progression and symptom severity [3].

\section{Conclusion}

Although there are many studies that briefly mention the plausible benefits of NAAG peptides on neurodegenerative diseases while using non-PD models, the results of those studies are directly translatable to PD's symptoms and pathology [13]. NAAG's downstream effects have the potential to reduce the loss of dopaminergic neurons, which have a primary role in the pathology and clinical manifestations in PD patients, since they reduce the release of glutamate and increase the release of neurotrophic factors as seen in other mechanisms that are neuroprotective of dopaminergic neurons [3,15-18]. Therefore, the use of NP inhibitors could decrease the degeneration of dopamine neurons which have a role in the primary pathophysiology of PD, since they decrease the release of glutamate and increase neuroprotection by increasing NAAG peptide levels and by preventing the hydrolysis of NAAG into glutamate $[3,12-15,17]$. This will allow the clinician to provide a better quality of life to PD patients. Future studies on PD-specific models of whether NP inhibitors that modulate mGlu3 receptors along with L-DOPA or other mGlu receptor modulators could provide potential therapeutic benefit may lead to new pharmacological interventions for PD patients.

In conclusion, the futility of some of the landmark trials using PD models and enrolling early stage PD patients to assess metabotropic glutamate receptor neuroprotection were unsuccessful due to the lack of tests and biomarkers to define the ideal stages of such intervention to take place. Future studies may elucidate the full effect of these modulator's neuroprotection by performing experiments on earlier stages of PD animal models, ideally prodromally [19].

\section{Acknowledgements}

None.

\section{Conflict of Interest}

No Conflict of Interest.

\section{References}

1. Xia N, Zhang Q, Ting Wang S, Gu L, Min Yang H, et al. (2015) Blockade of Metabotropic glutamate receptor 5 protects against DNA damage in a rotenone-induced Parkinson's disease model. Free Radical Biology and Medicine 89: 567-580.

2. Zhang Z, Zhang S, Fu O, Zhang Z, Lin K, et al. (2019) Roles of Glutamate Receptors in Parkinson's Disease. Int J Mol Sci 20(18): 4391.

3. Litim N, Morissette M, Di Paolo T (2017) Metabotropic glutamate receptors as therapeutic agents in Parkinson's disease: An update from the last 5 years of research. Neuropharmacology 115: 166-179.

4. Jankovic J (2008) Parkinson's disease: clinical features and diagnosis. J Neurol Neurosurg Psychiatry 79(4): 368-376.

5. Manuel Paz R, Tubert C, Monica Stahl A, Amarillo Y, Rela L, et al. (2021) Levodopa Causes Striatal Cholinergic Interneuron Burst- Pause Activity in Parkinsonian Mice. Movement Disorders.

6. Chen J, Swope D (2012) Pharmacotherapy for Parkinson's Disease. The Journal of Human Pharmacology and Drug Therapy 41(12P2): 161S $173 \mathrm{~S}$.

7. Block G, Liss C, Reines S, Irr J, Nibblelink D (1997) Comparison of Immediate-Release and Controlled Release Carbidopa/Levodopa in Parkinson's Disease. European Neurology 37: 23-27.

8. Meng X, Zhong J, Zeng C, K L Yung K, Zhang X, et al. (2021) MiR-30a-5p Regulates GLT-1 Function via a PKC $\alpha$-Mediated Ubiquitin Degradation Pathway in a Mouse Model of Parkinson's Disease. ACS Chem Neurosci 12(9): 1578-1592.

9. Bruno V, Battaglia G, Casabona G, Copani A, Caciagli F, et al. (1998) Neuroprotection by Glial Metabotropic Glutamate Receptors is Mediated by Transforming Growth Factor- $\beta$. The Journal of Neuroscience 18(23): 9594-9600.

10. Zhang W, Murakawa Y, M Woznik K, Slusher B, A F Sima A et al. (2006) The preventative and therapeutic effects of GCPII (NAALADase) inhibition on painful and sensory diabetic neuropathy. J Neurol Sci. 247(2): 217-223.

11. N Heale J, Bzdega T, Wroblewska B (2002) N-Acetylaspartylglutamate The Most Abundant Peptide Neurotransmitter in the Mammalian Central Nervous System. Journal of Neurochemistry 75(2): 443-452.

12. R Garrido Sanabria E, M Wozniak K, S Slusher B, Keller A, et al. (2004) GCPII (NAALADase) Inhibition Suppresses Mossy Fiber-CA3 Synaptic Neurotransmission by a Presynaptic Mechanism. J Neurophysiol. 91(1): 182-193.

13. Zhou J, H Neale J, G Pomper M, P Kozikowski A (2005) NAAG peptidase inhibitors and their potential for diagnosis and therapy. Nature Reviews Drug Discovery 4: 1015-1026.

14. Feng J, G Gurkoff G, C Van Ken, Song Minsoo, A Lowe David, et al. (2012) NAAG peptidase inhibitor reduces cellular damage in a model of TBI with secondary hypoxia. Brain Res 21(1469): 144-52.

15. Battaglia G, Molinaro G, Riozzi B, Marianna S, L Busceti C, et al. (2009) Activation of mGlu3 Receptors Stimulate the Production of GDNF in Striatal Neurons. PLoS One 4(8): e6591. 
16. G Thomas A, Bodner A, Ghadge G, P Roos R, S Slusher B (2009) GCP II inhibition rescues neurons from gp120IIIB-induced neurotoxicity. Journal of Neurovirology 15: 449-457.

17. Ambrosi G, Cerri S, Blandini F (2014) A further update on the role of excitotoxicity in the pathogenesis of Parkinson's Disease. J Neural Transm 121: 849-859.

18. Battaglia G, Riozzi B, Bucci D, Di Menna L, Molinaro G, et al. (2015) Activation of mGlu3 metabotropic glutamate receptors enhances GDNF and GLT-1 formation in the spinal cord and rescues motor neurons in the SOD-1 mouse model of amyotrophic lateral sclerosis. Neurobiology of Disease 74: 126-136.

19.J Masilamoni G, Smith Y (2018) Metabotropic glutamate receptors: Targets for neuroprotective therapies in Parkinson disease. Curr Opin Pharmacol 38: 72-80. 